Proceedings of the 2012 Winter Simulation Conference

C. Laroque, J. Himmelspach, R. Pasupathy, O. Rose, and A. M. Uhrmacher, eds.

\title{
HEALTH CARE LOGISTICS AND SPACE: ACCOUNTING FOR THE PHYSICAL BUILD ENVIRONMENT
}

\author{
Richard J. Boucherie \\ Erwin W. Hans \\ Timo Hartmann \\ University of Twente \\ P.O. Box 217 \\ 7523AE Enschede, THE NETHERLANDS
}

\begin{abstract}
Planning and scheduling of health care processes has improved considerably using operations research techniques. Besides analytical and optimization tools, a substantial amount of sophisticated discrete event simulation tools supporting (re-)design of existing logistical processes in and around hospitals has been developed. Surprisingly, these studies to a large extent consider a health care facility's physical configuration to be given and fixed (unchangeable). As layout has considerable influence on the facility's logistical performance (e.g., walking distance or transportation time of hospital beds), including layout in the optimization process seems to be a natural next step in further improving the possibilities to better plan and optimize health care processes. This paper illustrates the potential of accounting for building layout while using operational research optimization methods and discrete event simulation during the design of a new health care facility. To this end, we consider two developed discrete event simulation models of health care operations and evaluate how these two simulation models could benefit from a combination with building modeling methods to allow for the concurrent design of health processes and the buildings that need to host them. Based on this evaluation, the paper closes with a discussion of the benefits that such integration would offer.
\end{abstract}

\section{INTRODUCTION}

Health facilities not merely host health related activities, but also construct and constrain them (Prior 1988). The specific layout of a health facility influences health related processes and procedures and has been linked to noise levels, safety, staff fatigue (Ulrich 2006), and, of course, to the overall well-being of patients (Schweitzer, Gilpin, and Frampton 2004). The evidence for the influence of space on health processes is strong (Ulrich 2006) and it is by now generally accepted that the physical layout of health facilities is one of the main factors for the safe and efficient delivery of health services.

Despite the large body of existing evidence about the influence of architecture on health care design at large - Ulrich (2006) reported about more than 700 articles published before 2006 - the design of health facilities is still mainly based on an architect's personal imagination and intuition. At best, current architectural practice involves different stakeholders in so called participatory design activities, which allows members from other disciplines, such as doctors, nurses, hospital administrators, or even patients, to bring in their own intuition. In this design process, evidence in the form of historical data about health processes and procedural descriptions of health operations is seldom accounted for explicitly other than by using generalized architectural design or health process guidelines (see for example Malkin (1992) or Guenther and Vittori (2008)).

Operations management considers the design and organization of processes, such that they are both effective and efficient. Operations management has a rich history in industry and is included in the 


\section{Richard J. Boucherie, Erwin W. Hans, and Timo Hartmann}

architectural design, for example, during the design of production factories (Hopp and Spearman 2008). In contrast, although operations management has demonstrated its value for design of operational processes in existing health care facilities (Hall 2011), methods and research supporting its value for the design of health facilities are lacking. This is surprising, since, just like in industry, process improvement seems to be most effective in the design phase of facilities. For example, streamlining the room layout to host specific medical functions is best possible when designing the building, but only to a limited extent in existing buildings. Along the same lines, installations for medical gases, electricity, or data connections to support operational processes are best accounted for during initial building design. Later adjustments to buildings are either not possible or costly. Even worse, such later adjustments have been shown to have negative effects on patient safety (Bartley and Bjerke 2001). In summary, the seamless integration of operations management and architectural design has the great potential to enhance the quality of health facilities and health processes with respect to criteria, such as safety and efficiency.

To illustrate this potential, this position paper presents two discrete event simulation based studies that integrate process design while accounting for the specific layout of health care facilities. After introducing these studies as a context, this paper then presents our ideas on how to best integrate operations management methods with architectural design methods. The paper closes with a discussion of our ideas' impacts.

\section{SIMULATION OF HEALTH CARE PROCESSES}

Health care constitutes the largest industry in many developed countries, and managing it is a complex task due to its importance to society and the often politically charged atmosphere within which it exists (Vanberkel 2011). As a result, studies about operations management (OM) applications to support health care operations have been growing strongly in the last decade. This research has shown that OM techniques for modeling and optimizing processes are particularly useful in the health care context, where experimentation with the design and management of processes is not only costly and time consuming, but it can in fact be harmful for the patient.

Mathematical modeling and computer simulation allow prospective analyses of organizational interventions and process (re)design scenarios. Comparing various scenarios, robust conclusions about how to (re-)organize processes can be drawn that would not be possible using real life experimentation. While prior to 1999 simulation was not widely used as a tool for modeling complex integrated health care systems (Jun et al. 1999; Vanberkel et al. 2009), the literature on health care simulation and modeling is expanding at a rapid rate since then. This is nicely demonstrated in the structured literature review by (Brailsford et al. 2009).

For an exhaustive collection of examples of the application of OM methods to health care problems, we refer the interested reader to the online literature database ORchestra (Hulshof et al. 2011). 'ORchestra' categorizes applications by medical and OM technique. Categorized OM techniques applied in the studies collected in this database are, for example, numerical methods, such as discrete event, Monte Carlo, or system dynamics models, statistical methods, such as queuing models, and mathematical programming methods, such as integer linear programming. Additionally, OM based optimization techniques, such as (local search) heuristics or simulation based optimization, have been widely applied. Medical management fields in which these OM techniques have been implemented encompass medical planning, resource capacity planning, materials planning, and financial planning (Hans, Houdenhoven, and Hulshof 2012).

From these management areas, resource capacity planning and materials planning are of specific interest for the focus of this paper because these operations are highly dependent on the hosting building's layout. Resource capacity planning addresses the dimensioning, planning, scheduling, monitoring, and control of renewable resources, such as staff, equipment, and facilities. Materials planning addresses the acquisition, storage, distribution and retrieval of all nonrenewable (consumable) resources/materials, such as suture materials, blood, bandages, or food. 


\section{Richard J. Boucherie, Erwin W. Hans, and Timo Hartmann}

To provide an illustrative example of possibilities to improve these two areas with OM methods, the remainder of this section describes two simulations of health care operations we recently developed to resource capacity and materials planning problems.

\subsection{Designing an Outpatient Clinic's Physical Lay-out Based on Managerial Performance Targets}

A major first entry point for patients is the outpatient clinic. The outpatient clinic typically consists of many consultation rooms and waiting rooms. The structure of the process or policy in which doctors see patients is of considerable importance for the design of the clinic. Traditionally, in outpatient clinics the doctor remains in a consultation room while patients stay in the waiting room until the start of the consultation, we call this the Patient-to-Doctor (PtD) policy. This policy requires a large waiting room and the number of consultation rooms coincides with the number of doctors. An alternative approach is the Doctor-to-Patient (DtP) policy, whereby doctors travel between multiple consultation rooms, in which patients prepare for their consultation and doctors visit patients after they have completed preparation. Under this policy, the number of consultation rooms is larger than the number of doctors, and the waiting room can be kept much smaller. Under the DtP policy, doctors save time by consulting fully prepared patients, but lose time by traveling among consultation rooms. Comparison of these policies in a queuing theoretic framework via both analytical methods and a discrete-event simulation approach has revealed a clear switching curve between the policies that, indeed, is fully characterized by the doctors traveling time and the patients preparation time (Hulshof, Vanberkel, Boucherie, Hans, van Houdenhoven, and van Ommeren 2012). In both frameworks, the required capacity, e.g., number of consultation rooms in the Doctor-to-Patient policy and size of the waiting room in both policies can be obtained given managerial performance targets such as patients waiting time and the time the patient spends in the clinic. These results have been applied in a medium-sized general hospital in the Netherlands to redesign their outpatient clinics based on historical data. In particular, these results provided quantitative measures and formal proof to support switching the operation of the outpatient clinic from a PtD-policy to a DtP-policy, including the required number of consultation rooms for each doctor under the DtP-policy. Designing outpatient clinics' physical lay-out does not only require a trade-off between these policies at the managerial level, that basically matches available staff and patient needs, but also should take into account the actual (available) volume and floor plan of the clinic. This is the next step in the design process of hospitals.

\subsection{Designing an Integrated Emergency Post}

A second major entry point for patients is the emergency department. A trend in the Netherlands is to integrate the Emergency Department (ED) with a General Practitioners post (GP post) into an Integrated Emergency Post (IEP) to allow all patients to enter via the same entry point, while being able to discriminate among patients with different required medical care levels. Designing an integrated emergency post, in which patients receive optimal care, with optimal use of resources and without unnecessary delays, while taking patient preferences into account, requires simultaneous consideration of the entry point's physical lay-out and logistical and medical processes. To this end, a conceptual model of the IEP was created (Visser 2011) that is sufficiently flexible to allow for modification of the physical lay-out, patient routes, and distributions for, e.g., number of arriving patients, type of patients and treatment times. Subsequently, the model was coded in a discrete event simulation model. The discrete event model's verification and validation requires actual historical data (Visser 2011). To this end, the recently opened IEP in the city of Almelo in the Netherlands was considered as test case. This IEP was opened in April 2010 as collaboration between the GP post Almelo and the ED of the hospital ZGT Almelo that both have sufficient historical data available for determining the input data for the simulation model and for validating and verifying the model using the various techniques described by (Law and Kelton 2000). 


\section{Richard J. Boucherie, Erwin W. Hans, and Timo Hartmann}

Both illustrative examples show a high dependency of the process on practitioners' and patients' walking routes and walking times that were obtained from historical data. Walking routes are defined by the building's layout. Therefore accounting for these routes already in the design phase of the building would have allowed to further streamline the above processes. The first example shows a switching curve between where a Patient-to-Doctor (PtD) policy becomes more efficient than a Doctor-to-Patient (DtP) policy. This curve is defined by the time a doctor needs to travel to the patients. Further optimization of travel times will even further increase the DtP strategy's efficiency.

Similar considerations hold for the second case. Using the discrete event simulation, not only different organizational interventions can be tested, but also the influence of different layouts on the physical and spatial configuration of the Integrated Emergency Post (IEP). For different layouts of the IEP the managerial performance measures can be evaluated with the developed discrete event simulation. Different designs involve different patient flows between the GP and ED part of the IEP. Along the same lines, the operational performance would vary according to different waiting locations for patients, such as common waiting areas or an additional waiting room for patients waiting for diagnosis results. The current simulation models provide a good starting point for evaluating not only the operational performance, but also the effects of different spatial and physical designs Optimization for both lay-out and performance simultaneously requires an integrated approach. In the next section, we propose a framework to allow for such an integrated approach.

\section{ACCOUNTING FOR THE BUILDING: A DESIGN PROCESS FRAMEWORK FOR INTEGRATED ARCHITECTURAL AND MEDICAL OPERATIONS DESIGN ACTIVITIES}

Our proposed framework for the integrated design of health operations and architectural layout is depicted in Figure 1. The framework is based on the idea of health care service design, in which a health care service is comprised of a combination of several atomic medical functions. An example for a health care service design is the above described Integrated Emergency Post, where all patients first see a general practitioner (first atomic function) and then, according to the practitioner's first assessment are redirected to specific consecutive functions, such a X-ray screening, laboratory services, or medical specialist consultations. Based on the identification of the required atomic functions for the health service, a list can be developed with requirements for each atomic medical function and the required area. This list can then be transferred into an architectural room book that describes the required spaces to host the envisioned health services. Combining the information about the required/offered health care services and the information from the room book, a spatial layout can then be established that allocates the different required spaces within a floor plan and establishes connections between the spaces by designing, for example, corridors and hallways and by locating doors and access ways.

For new hospital design the natural process to proceed through the outlined steps would be in the above used order: design a number of health services to be delivered, design the required atomic medical functions, design the architectural room book, and finally design the spatial layout. However, most health service design activities nowadays are concerned with the redesign of existing facilities and services. Therefore, the starting point and how to iterate between the different steps should be considered on a case-by-case basis. For example, to design the renovation of an existing hospital it might be valuable to first gain an understanding of the existing space in the form of a room book and layout. In this case, the architectural information would then drive decisions about what health functions and atomic services actually can be hosted within the constraints of the existing facility. We have indicated this mutual influence between the different parts through double arrows in Figure 1.

Once developed, the above scenarios can then be modeled with a combination of operations management modeling methods and building modeling methods (Eastman et al. 2008; Hartmann et al. 2008). The resulting integrated models could then serve as an input to methods that allow for the automated checking of constraints not primarily related to the efficiency and effectiveness of health processes, for example on existing safety regulations or building codes. Process efficiency and risk indicators for these integrated 
Richard J. Boucherie, Erwin W. Hans, and Timo Hartmann

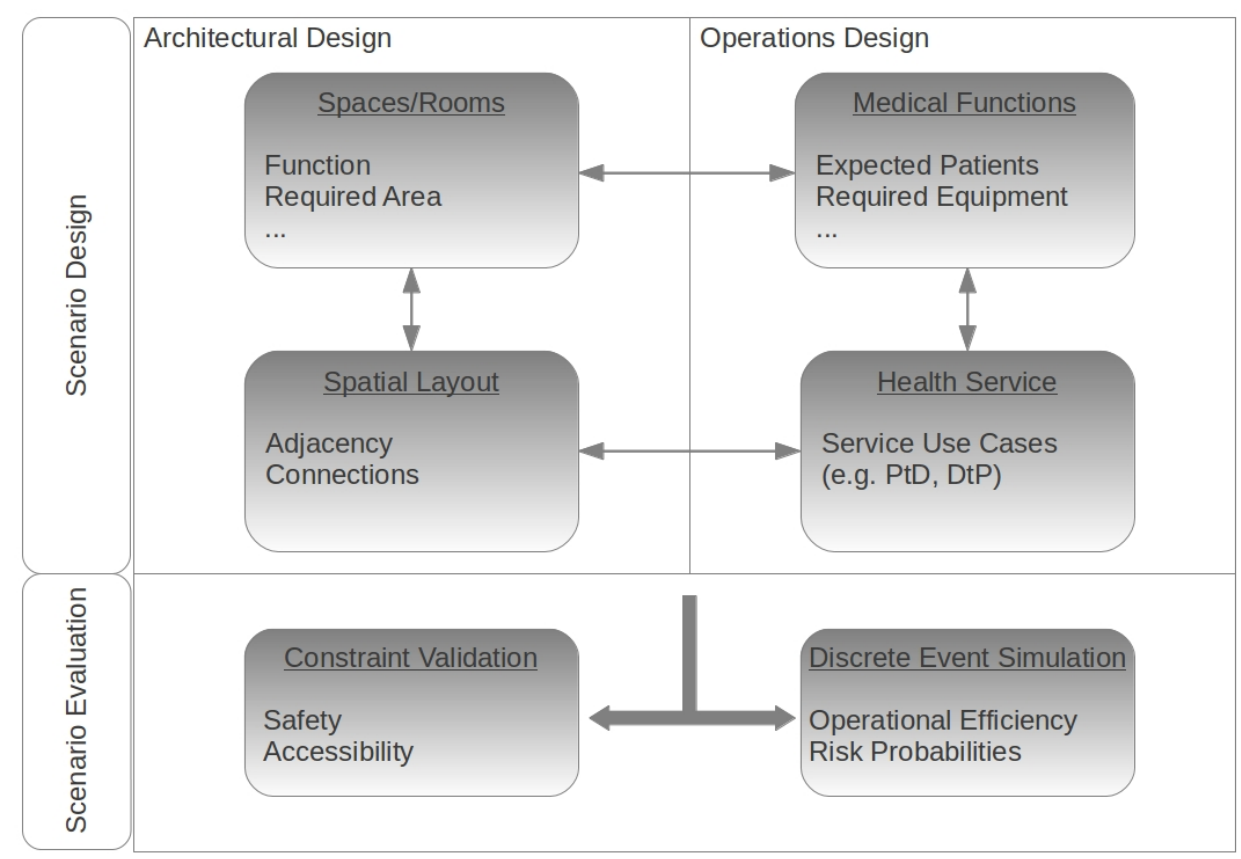

Figure 1: Process framework to support the integration of operations management and architectural design of a health care facility.

models can be evaluated using discrete-event simulation. Based on the outcomes of this evaluation, several operational or architectural aspects of the scenario can then be adjusted in an effort to jointly streamline the health care facility's and the health service's overall design.

\section{DISCUSSION}

Operations management has the potential to improve the architectural design of health facilities. In current practice, an architect is provided with general design requirements for the facility to be designed, only. These requirements are traditionally defined in the form of spatial requirements that have to be accounted for while laying out the facility. Moving beyond this practice, there is currently a shift towards the provisioning of function based requirements instead of clearly specified spatial requirements. Hence, instead of providing an architect with a clear specification about the spatial requirements, architects find themselves more and more in the role of deriving the spatial requirements themselves. In this practice, the initial requirements for a facility to support specific health services are vague and often not fully understood by the architect, which may lead to sub-optimal design. Moreover, due to the detachment of operational planning activities and architectural design, problems with the layout are often only realized quite late in the construction process. Changes in later stages unfortunately are quite costly and in the past often have lead to unaccounted cost overruns. Even worse, facilities were constructed and delivered that were not well suited to provide the required functionality to deliver the planned health services.

The framework sketched in Figure 1 with its explicit mapping between functional and operational requirements and spatial layout has the potential to improve current architectural practice significantly. It will give architects insight into the functional requirements, which, in turn, allows them to improve layouts. Integrating automatic constraint-checking and performance analysis of health processes, architects will also get direct feedback about the feasibility and optimality of a certain layout alternatives. These may include additional constraints with respect to building codes, such as fire safety or structural safety rules from the 


\section{Richard J. Boucherie, Erwin W. Hans, and Timo Hartmann}

knowledge domain of the architect or knowledge from operations managers, such as how many patients can be maximally allowed within a certain space. All in all, we anticipate that architectural design supported by the presented framework will not only deliver layouts with less process inhibiting errors, but layouts that streamline the health services they should support.

The two simulation examples we described barely scratch the surface of the possibilities for improving organizational processes in health care, and its integration into architectural choices while building, rebuilding, or expanding health facilities. As health care expenditures are under much pressure and technology becomes increasingly complex, efficient utilization of (new) facilities is a design constraint. In particular expensive resources like (minimal invasive) operating theaters, radiation therapy and radio-diagnostic facilities, and intensive care units should be located such that optimal use is guaranteed. Furthermore, given the inherent variable health care demand, the design should strive for optimal flexibility, to allow shared use of facilities like wards, consultation rooms, and waiting rooms. Scenario analyses by simulation techniques can support the architects to achieve this.

The presented framework not only streamlines the separate design efforts of operational managers and architects in isolation, it also supports collaborative design by bringing together representatives from both disciplines (architects and managers) in design workshops. With the explicit link between both fields discrete event based layout evaluation schemes could be used as boundary spanners. Architects will be able to communicate their knowledge with respect to a specific building layout, while operations managers may share their knowledge about the health operations. Fast scenario evaluation will then allow discriminating among various operational and layout alternatives and reach conclusions in single workshop sessions.

We are aware that the above sketched design process framework can already be supported by many of the existing operations research and simulation tools. Existing commercial solutions, for example, allow for modeling of walking distances or space requirements. Nevertheless, existing methods and tools used in architectural practice do not integrate with them. Hence, in current practice architects and process designers cannot work well together to concurrently design health facilities and health processes. To allow for a more integrated practice, much work is required developing tools and methods for integrating these two areas of design. This work will not only require a more close understanding of the differences in simulating and designing health care processes and facilities versus the simulation of processes and facilities in manufacturing for which most of the existing tools mainly have been developed for. The work will also require an increase in mutual understanding between the two different work cultures of operation researchers and architects, accounting for differences in objectives, tools used, and processes followed. The presented process framework in this position paper intends to provide a first stepping stone to trigger more research to understand differences and to derive requirements for the methods and tools specific to the design of health care processes and architecture and a close integration of these two design fields.

\section{CONCLUSION}

In this position paper, we argue for the need to better integrate the fields of operations management and architecture for the design of health processes and the facilities that should host these processes. Based on two examples illustrating the operational redevelopment of two health service operations using discrete event simulation techniques, we underscore this argument by showing that both operations are intrinsically dependent on the architectural spatial layout of the buildings. To provide first ideas of how to establish this integration, the paper has introduced a framework to support the integrated design or redesign of health facilities. We anticipate that this paper and the framework presented can provide a first stepping-stone towards multi-disciplinary design of our future health care systems.

\section{ACKNOWLEDGMENTS}

This research is partly founded by the Province of Overijssel and the institute for biomedical technology and technical medicine (MIRA) of the University of Twente. The paper is written in a joint collaboration 


\section{Richard J. Boucherie, Erwin W. Hans, and Timo Hartmann}

between researchers of the University of Twente from the stochastic operations research group, the center for health care operations improvement and research, and the construction engineering and management department's center for visualization and simulation in construction. We would like to acknowledge the two hospitals that supported the two operation research efforts presented in this paper: ZGT in Almelo and RIVAS Zorggroep.

\section{REFERENCES}

Bartley, J., and N. Bjerke. 2001. "Infection control considerations in critical care unit design and construction: a systematic risk assessment". Critical Care Nursing Quarterly 24 (3): 43.

Brailsford, S., P. Harper, B. Patel, and M. Pitt. 2009. "An analysis of the academic literature on simulation and modelling in health care". Journal of Simulation 3 (3): 130-140.

Eastman, C., P. Teicholz, R. Sacks, and K. Liston. 2008. BIM handbook. Wiley Online Library.

Guenther, R., and G. Vittori. 2008. Sustainable healthcare architecture. Wiley.

Hall, R. 2011. Handbook of Healthcare System Scheduling, Volume 168. Springer Verlag.

Hans, E., M. Houdenhoven, and P. Hulshof. 2012. "A Framework for Healthcare Planning and Control". Handbook of Healthcare System Scheduling:303-320.

Hartmann, T., J. Gao, and M. Fischer. 2008. "Areas of application for 3D and 4D models on construction projects". Journal of Construction Engineering and management 134:776.

Hopp, W., and M. Spearman. 2008. Factory physics. McGraw-Hill Irwin Irwin.

Hulshof, P., R. Boucherie, J. Essen, E. Hans, J. Hurink, N. Kortbeek, N. Litvak, P. Vanberkel, E. Veen, B. Veltman et al. 2011. "ORchestra: an online reference database of OR/MS literature in health care". Health care management science 14:383-384.

Hulshof, P., P. Vanberkel, R. Boucherie, E. Hans, M. van Houdenhoven, and J. van Ommeren. 2012. "Analytical models to determine room requirements in outpatient clinics". OR spectrum 34:391-405.

Jun, J., S. Jacobson, J. Swisher et al. 1999. "Application of discrete-event simulation in health care clinics: A survey". Journal of the operational research society 50 (2): 109-123.

Law, A., and D. Kelton. 2000. "Simulation modeling and analysis".

Malkin, J. 1992. Hospital interior architecture: Creating healing environments for special patient populations. Van Nostrand Reinhold.

Prior, L. 1988. "The architecture of the hospital: a study of spatial organization and medical knowledge". British Journal of Sociology 39:86-113.

Schweitzer, M., L. Gilpin, and S. Frampton. 2004. "Healing spaces: elements of environmental design that make an impact on health". Journal of Alternative \& Complementary Medicine 10 (Supplement 1): 71-83.

Ulrich, R. 2006. "Essay: Evidence-based health-care architecture". Lancet 368 (1): 538-539.

Vanberkel, P., R. Boucherie, E. Hans, J. Hurink, and N. Litvak. 2009. "A survey of health care models that encompass multiple departments".

Vanberkel, P. T. 2011, May. Interacting hospital departments and uncertain patient flows : theoretical models and applications. Ph. D. thesis, University of Twente, Enschede, the Netherlands.

R.E. Visser 2011, December. "Modeling an integrated emergency post: designing a simulation model for the collaboration between the GP post Almelo and the emergency department of ZGT Almelo".

\section{AUTHOR BIOGRAPHIES}

RICHARD J. BOUCHERIE is Professor of Stochastic Operations Research at the Departement of Applied Mathematics of the University of Twente, The Netherlands. His email address is r.j.boucherie@utwente.nl.

ERWIN W. HANS is Associate Professor for Operations Management and Process Optimization in Health Care at the School of Management and Governance of the University of Twente. His email address is 
Richard J. Boucherie, Erwin W. Hans, and Timo Hartmann

e.w.hans@utwente.nl.

TIMO HARTMANN is Associate Professor for Visualization and Simulation in Construction at the Faculty of Engineering Technology of the University of Twente. His email address is t.hartmann@utwente.nl. 